\title{
Modelagem nos anos iniciais da educação básica: como os estudantes modelam situações-problema?
}

\author{
Modeling in the early years of basic education: \\ as students model problem-situations?
}

\begin{abstract}
Morgana Scheller ${ }^{1}$. Danusa de Lara Bonotto ${ }^{2}$. Zulma Elizabete de Freitas Madruga ${ }^{3}$. Maria Salett Biembengut ${ }^{4}$. Jose Maria Chamoso Sánchez ${ }^{5}$
\end{abstract}

\begin{abstract}
Resumo: Neste artigo objetiva-se compreender e analisar como estudantes dos Anos Iniciais da Educação Básica, em atividade de Modelagem, resolvem situações-problema que podem requerer domínio algébrico simbólico e que linguagens fazem uso na expressão dos modelos. Os dados empíricos advieram de uma das práticas de Modelagem desenvolvidas com 16 estudantes de escola pública durante 11 horas/aula, no período de junho e julho/2015 e foram obtidos dos materiais produzidos pelos estudantes, gravações de áudio e vídeo e observação realizada durante a prática sobre a numeração do calçado de uma pessoa. No processo os estudantes propuseram modelo estabelecendo relações numéricas entre as variáveis, obtendo generalização e realizando previsões. A análise evidenciou que eles resolvem situação-problema apresentando modelos nas linguagens natural, numérica e tabular, evidenciando domínio algébrico característico do pensamento algébrico anterior à linguagem simbólica, pertinente ao nível de escolaridade, e extrapolam objetivos referentes a realização de previsão a partir do modelo.
\end{abstract}

Palavras-chave: Educação básica. Modelagem na educação. Ensino e pesquisa. Álgebra.

\begin{abstract}
This article aims to understand and analyze how elementary students solve problems through modeling, which may require symbolic algebra and languages to use the model's expression. The empirical data have been constructed through one of the modeling practices developed with 16 public school students for 11 hours / class, between June and July / 2015. It were obtained from materials produced by students, audio and video recordings and observation during practice on the numbers of a person's shoes. In the modeling process, the students proposed model establishing numerical relationships between variables, obtaining generalizations and making forecasts. The analysis showed that they solve the problem situation by presenting models in natural languages, in numerical and tabular formats demonstrating characteristic algebra from previous algebraic thought to the symbolic language, appropriate to the educational level, and go beyond the objectives regarding the completion of the forecast from model.
\end{abstract}

Keywords: Modeling in Education. Elementary school. Teaching and research. Algebra.

\footnotetext{
${ }^{1}$ Instituto Federal de Educação, Ciência e Tecnologia Catarinense (IFC), Rio do Sul, SC, Brasil. E-mail: $<$ morganascheller@yahoo.com.br>.

${ }^{2}$ Universidade Federal da Fronteira Sul, Cerro Largo, RS, Brasil.

${ }^{3}$ Secretaria Estadual de Educação do Rio Grande do Sul, Campo Bom, RS, Brasil.

${ }^{4}$ Pontifícia Universidade Católica do Rio Grande do Sul (PUCRS), Porto Alegre, RS, Brasil.

${ }^{5}$ Universidad de Salamanca, Facultad de Educación, Departamento de Didáctica de las Matemáticas y de las Ciencias Experimentales, Salamanca, España.
} 


\section{Introdução}

O processo de ensino e de aprendizagem orientado pela pesquisa "instiga o estudante no sentido da curiosidade em direção ao mundo que o cerca, gera inquietude, possibilitando que o estudante possa ser protagonista na busca de informações e de saberes, quer sejam do senso comum, escolares ou científicos" (BRASIL, 2013, p. 164). Neste sentido, requer um método que propicie ao estudante aprender matemática e, ao mesmo tempo, aprender a fazer pesquisa. Isto é: entender uma situação-problema e dominar as linguagens necessárias para descrevê-la, representá-la, resolvê-la e interpretá-la no contexto de sua existência. Dentre as propostas pedagógicas para o ensino de matemática, a que traz em essência este processo é a Modelagem na Educação - Modelação, definida como um método de ensino com pesquisa na instituição escolar, nos limites da sala de aula (BIEMBENGUT, 2004, 2014).

A Modelagem na Educação possibilita ao estudante aprender Matemática e Ciências de modo integrado às diversas áreas do conhecimento, em especial, àquela de interesse. Práticas de Modelagem diferem das habituais atividades em que os estudantes desenvolvem em sala de aula, pois envolvem situações-problema autênticas que precisam ser interpretadas e descritas em representações matemáticas (ENGLISH; WATTERS, 2004b). Nesse sentido, desenvolver práticas de Modelagem com estudantes já nos Anos Iniciais da Educação Básica, e a partir da própria coleta de dados, pode contribuir para a ativa participação de todos no processo de aprendizagem e, de acordo com English (2013), para o entendimento da importância da matemática e a sua presença em situações variadas, e consequentemente, contato com linguagem e pensamento científico. Estudos indicam que, independente de qual componente curricular é explorado, a pesquisa deve estar presente desde o início da vida escolar, não sendo atribuição apenas da Educação Matemática.

As pesquisas em Modelagem apresentam-se, em vasto número, no cenário da Educação Matemática. Esta, em grande parte, tem seus estudos com foco no Ensino Superior e Formação de Professores, sendo incipientes aquelas cujo foco centra-se nos Anos Iniciais da Educação Básica como destacam Almeida e Tortola (2014); English (2010); English e Sriraman (2010); Silva e Klüber (2012), Souza e Luna (2014). Dentre as identificadas, destacam-se nesta pesquisa, além destes, os estudos de Biembengut (2007); English (2010, 2013); English e Watters (2004a, 2004b); Luna e Alves (2007); Luna, Souza e Lima (2015); Luna, Souza e Santiago (2009); National Council of Teachers of Mathematics (2000); Souza, Luna e Lima (2014); Tortola (2012); Tortola e Almeida (2013).

Dessas pesquisas cujas fontes são estudantes dos Anos Iniciais, English e Watters (2004a, 2004b) e English (2010) apresentam resultados de programa desenvolvido na Austrália com estudantes de quatro classes de terceira série na resolução de problemas, e evidenciam que os alunos expressam por meio de relatório (uma situação) e por uso de carta (outra situação contendo desenhos) suas percepções a respeito da solução do problema, argumentando as devidas recomendações. Demonstram, ainda, que os estudantes não permanecem em mero levantamento de dados. Luna, Souza e Santiago (2009) concluem que estudantes do quinto ano do Ensino Fundamental percebem a presença dos modelos matemáticos na sociedade e suas implicações sociais, políticas e econômicas em diversos segmentos da sociedade; contudo, não foram passivos ou resistentes a esse tipo de atividade, fato em que os autores identificaram em outras pesquisas no nível. Embora os modelos proporcionaram a resolução do problema, 
neste estudo não há indícios de relação com pensamento algébrico. O estudo de Tortola (2012) confirmou que diferentes modelos matemáticos são elaborados por estudantes do quarto ano e, devido ao uso que fazem da linguagem, os alunos têm características específicas ao nível de escolaridade. Almeida e Tortola (2014) ao investigarem como estudantes do quarto ano utilizam a linguagem nas atividades de Modelagem Matemática, identificaram que os usos da linguagem subsidiam as ações dos estudantes nas referidas atividades, assim viabilizando a produção dos modelos matemáticos (linguagens natural e numérica) para solução da situação-problema proposta. No entanto, estas pesquisas sobre Modelagem não destacam como os estudantes dos Anos Iniciais da Educação Básica resolvem situações-problema que podem requerer domínio algébrico formal (linguagem simbólica), tampouco que modelos podem utilizar neste caso.

Vale destacar que nos anos de 1980, quando a Modelagem passa a ser mais amiúde defendida como processo ou método de ensino na Educação Básica, em particular no Ensino Médio, acreditava-se ser o mais adequado, pois tais estudantes já possuiriam os domínios aritmético, algébrico e geométrico. No entanto, vários estudos mostraram que o trabalho com a Modelagem pode ser exitoso desde os Anos Iniciais, tais como: Biembengut (2007); Carpenter e Romberg (2004); English (2006); English e Watters (2004a, 2004b).

Esses pesquisadores sinalizaram que os estudantes dos Anos Iniciais são capazes de desenvolver seus próprios modelos e sistemas significativos para lidar com complexas situações -problema. Entretanto, não apresentam resultados quanto ao domínio algébrico destes estudantes. Segundo Blanton e Kaput (2005); Fiorentini, Fernandes e Cristóvão (2005); Fiorentini, Miorin e Miguel (1993) e Kieran (2004), dentre outros, estudantes dos Anos Iniciais do Ensino Fundamental mesmo não tendo condições de estruturar a linguagem algébrica simbólica, possuem condições de desenvolvimento do pensamento algébrico. Assim, como estes estudantes resolvem situações-problema utilizando Modelagem quando os modelos podem requerer dominio algébrico simbólico? Neste caso, como expressam estes modelos, quer dizer, que linguagens fazem uso na expressão dos modelos? Estas questões impulsionaram a presente pesquisa: compreender e analisar como estudantes dos Anos Iniciais resolvem situações-problema de modelagem que podem requerer domínio algébrico simbólico e que linguagens utilizam na expressão de modelos neste processo.

A partir dos resultados prévios de pesquisas realizadas com estudantes dos Anos Iniciais, supõe-se que, em práticas de Modelagem na Educação, os estudantes resolvam situações-problema com linguagem pertinente ao nível de escolaridade e, para tal, elaboram modelo utilizando-se de diferentes registros de representação sendo inclusive capazes de realizarem inferências sobre os mesmos, extrapolando as competências matemáticas exigidas para o referido nível.

\section{Marco Teórico - O Pensamento Algébrico}

Nos Parâmetros Curriculares Nacionais (PCN) (BRASIL, 1998, p. 115) consta que o estudo da Álgebra "constitui um espaço bastante significativo para que o aluno desenvolva e exercite sua capacidade de abstração e generalização, além de lhe possibilitar a aquisição de uma poderosa ferramenta para resolver problemas"; conduz para a expressão de generalizações sobre regularidades observadas em algumas sequências numéricas. Porém, tais referências dizem respeito aos Anos Finais do Ensino Fundamental, não sendo encontrada menção sobre o estudo da Álgebra para os Anos Iniciais. Em Brasil (1997), tem-se apenas destacado que neste 
nível de ensino já é possível o desenvolvimento de uma "pré-álgebra", porém sem defini-la, a qual deve ser ampliada nos Anos Finais do Ensino Fundamental. Apenas em 2012 que um documento oficial traz a orientação sobre o pensamento geométrico, porém apenas para o ciclo de alfabetização $\left(1^{\circ}\right.$ ao $3^{\circ}$ do Ensino Fundamental) (BRASIL, 2012).

De acordo com National Council of Teacher of Mathematics $(2000)^{6}$, a álgebra precisa estar em um contínuo curricular na Educação Básica, uma vez que contribui ao estudante base sólida ao pensamento algébrico. Assim, o estudante dos Anos Iniciais, ao analisar e refletir a respeito de como quantidades se relacionam e de representar situações matemáticas utilizando-se de objetos, figuras, esquemas e símbolos, experimenta atividades no campo das relações funcionais e da modelagem matemática favoráveis ao desenvolvimento algébrico. Isto é possível quando incentivado a utilizar os vários tipos de representação de ideias e relações matemáticas, como linguagem natural, tabelas e gráficos (CARRAHER; SCHLIEMANN, 2007). Decorre daí a sugestão para a integração entre os conhecimentos aritméticos e algébricos, a geometria e a análise dos dados e a estatística. Para o National Council of Teacher of Mathematics (2007, p. 20), todos estes apontamentos serão possíveis se os estudantes estiverem envolvidos em atividades matemáticas significativas para a construção de conceitos. Atividades estas pertencentes ao seu contexto e conduzidas de modo que "provoquem interrogações, possuindo um nível de desafio que convide à especulação e ao trabalho árduo".

O National Council of Teacher of Mathematics (2007) sugere normas para o ensino de Álgebra visando o desenvolvimento do pensamento algébrico para cada nível de escolaridade e, também, objetivos diferenciados. No Quadro 1 ilustram-se normas para o Ensino de Álgebra relativas à $2^{\mathrm{a}}$ parte (ciclo) do Ensino Fundamental. Após a publicação em 2000, em relação aos Anos Iniciais, vários trabalhos em todo o mundo foram desenvolvidos sobre a abordagem do desenvolvimento algébrico, como os de Canavarro (2007); Carraher, Martinez e Schliemann (2008); Carraher, Schliemann e Brizuela (2001); Kaput, Carraher e Blanton (2008); Kieran (2004); Schliemann, Carraher e Brizuela (2007) e Vale et al. (2007). No Brasil, entre outros estão os de Falcão (2003); Gomes (2003); Luna e Souza (2013) e Silva e Savioli (2012).

\footnotetext{
${ }^{6}$ Traduzido e editado em 2007 pela Associação de Professores de Matemática (APM) de Portugal como Princípios e Normas para a Matemática Escolar, é um documento que serve de referência, orientação e recurso para todos aqueles cujas decisões afetam a educação matemática dos estudantes da Educação Básica (Pré-escolar ao $12^{\circ}$ ano), em particular, professores, responsáveis pela elaboração dos currículos, formadores e decisores de políticas de educação matemática.
} 
Quadro 1. Normas para o Ensino de Álgebra para $3^{\circ}$ ao $5^{\circ}$ anos de escolaridade

\begin{tabular}{|l|l|}
\hline \multicolumn{1}{|c|}{ Itens } & \multicolumn{1}{|c|}{ Objetivos para $\mathbf{3}^{\mathbf{0}}$ ao $\mathbf{5}^{\mathbf{0}}$ anos de escolaridade } \\
\hline $\begin{array}{l}\text { Compreender padrões, } \\
\text { relações e funções }\end{array}$ & $\begin{array}{l}\text {. Descrever, ampliar e fazer generalizações acerca de padrões } \\
\text { geométricos e numéricos; } \\
\text {. Representar e analisar padrões e funções, usando palavras, tabelas } \\
\text { e gráficos; }\end{array}$ \\
\hline $\begin{array}{l}\text { Representar e analisar } \\
\text { situações e estruturas } \\
\text { matemáticas usando símbolos } \\
\text { algébricos }\end{array}$ & $\begin{array}{l}\text {. Identificar propriedades como a comutatividade, a associatividade } \\
\text { e distributividade e aplicá-las no cálculo com números inteiros; } \\
\text {. Representar a ideia de variável como quantidade desconhecida, } \\
\text { através de uma letra ou símbolo; } \\
\text {. Expressar relações matemáticas usando equações (valor } \\
\text { desconhecido); }\end{array}$ \\
\hline $\begin{array}{l}\text { Usar modelos matemáticos } \\
\text { para representar e } \\
\text { compreender relações } \\
\text { quantitativas }\end{array}$ & $\begin{array}{l}\text {. Modelar situações problemáticas, usando objetos e recorrer } \\
\text { a representações como gráficos, tabelas e equações para tirar } \\
\text { conclusões; }\end{array}$ \\
\hline $\begin{array}{l}\text { Analisar a mudança (variação) } \\
\text { em vários contextos }\end{array}$ & $\begin{array}{l}\text {. Investigar a forma como a mudança numa variável se relaciona } \\
\text { com a mudança de uma segunda variável; } \\
\text {. Identificar e descrever situações com taxas de variação constantes } \\
\text { ou variáveis e compará-las. }\end{array}$ \\
\hline
\end{tabular}

Fonte: National Council of Teacher of Mathematics (2007).

Esses estudos e também os de Fiorentini, Miorin e Miguel (1993); e Lins e Gimenez (1997), apontam para a importância do desenvolvimento algébrico desde os Anos Iniciais da Educação Básica e ainda, que tal desenvolvimento seja simultâneo ao aritmético. O conceito é defendido por Blanton e Kaput (2005); Carraher e Schliemann (2007); Kieran (2004) como Early Algebra (EA), cujo suporte apoia-se na concepção de sinais de reconhecimento de álgebra do pensamento algébrico em atividades de matemática na escola elementar (6 a 12 anos). Nesta perspectiva, o pensamento algébrico refere-se ao "processo pelo qual os estudantes generalizam ideias matemáticas a partir de um conjunto de casos particulares, estabelecem essas generalizações através de discurso argumentativo, e expressam-nas de formas progressivamente mais formais e adequadas à sua idade" (BLANTON; KAPUT, 2005, p. 413). Para Kieran (2004, p. 149, tradução nossa), o pensamento algébrico nos Anos Iniciais

[...] envolve o desenvolvimento de formas de pensar no âmbito das atividades para as quais a linguagem simbólica pode ser usada como uma ferramenta, mas que não são exclusivas para álgebra e com as quais podem se envolver sem usar qualquer linguagem simbólica, tais como analisar relações entre quantidades, observar a estrutura, estudar variações, generalizar, resolver problemas, modelar, justificar, provar e prever.

De acordo com Bednarz e Janvier (1996), Blanton e Kaput (2005), Carraher e Schliemann (2007), como perspectivas à iniciação a álgebra no ensino elementar ou EA (pensamento 
algébrico) estão a generalização da aritmética, a resolução de problemas, pensamento funcional e a modelagem matemática. Em relação à modelagem, com referência à aprendizagem de álgebra escolar, "o ponto crucial do processo é a fase de formulação que resulta na criação do modelo (expressão simbólica, gráfico, tabela de valores, entre outros) na base de hipóteses" (BEDNARZ; JANVIER, 1996, p. 10).

\section{Procedimentos Metodológicos}

Esta pesquisa aplicada, sob a orientação das questões apontadas na apresentação, desenvolveu-se em uma perspectiva qualitativa ${ }^{7}$ (BOGDAN; BIKLEN, 1994; DENZIN; LINCOLN, 1994) e descritiva interpretativa, na busca de compreender detalhadamente os significados e características das situações apresentadas pelos estudantes, colaboradores desta investigação. Este tipo de pesquisa busca a compreensão dos dados gerados em seu ambiente natural, focada no discurso oriundo deste espaço.

A pesquisa foi desenvolvida em uma Escola Pública Municipal de Ensino Fundamental da Educação Básica do Estado de Santa Catarina que oferta ensino normal, nos turnos matutino e vespertino. Neste espaço há desenvolvimento de algumas ações educativas, como desenvolvimento de projetos ambientais relacionados ao descarte de óleo vegetal de cozinha e reciclagem de materiais. O trabalho em classe ocorre, em sua maior parte, com livro, atividades e exposição oral. De acordo com a professora da turma, na Escola, esses estudantes não haviam tido contato com Modelagem na experiência escolar.

Participaram de todo o desenvolvimento das práticas 16 estudantes (oito meninos e oito meninas) do $4^{\circ}$ Ano do Ensino Fundamental, período vespertino, com idades entre nove e dez anos, entre os meses de maio a julho de 2015, durante 33 horas/aulas (de três a cinco aulas semanais). Destaca-se que apenas um estudante era 'repetente' no referido ano escolar. A turma, nas práticas de Modelagem, foi conduzida por um dos autores desta pesquisa e assistida pela professora regente da turma. Considera-se que o contato direto do pesquisador com a situação a ser investigada é relevante. Neste período, as três práticas de Modelagem na Educação foram desenvolvidas e elegeu-se para esta pesquisa o episódio referente a numeração do calçado de uma pessoa, terceira prática (11 horas/aula), ao qual possibilitou a exploração de conceitos de medidas, tratamento de informação (tabelas, gráficos e quadros) e números decimais. A prática foi desenvolvida em três encontros de três horas/aulas por semana e outro de duas horas/aula.

Diante da defesa de Biembengut (2004), English e Watters (2004a, 2004b), Maaß (2005) e English (2010), de que a Modelagem já pode e deve ser inserida nos Anos Iniciais da Educação Básica, a prática de Modelagem na Educação numeração do calçado pautou-se na concepção de Biembengut (2014) e foi desenvolvida com base nas três fases:

\footnotetext{
${ }^{7}$ De acordo com os autores, compreende-se aqui que na pesquisa qualitativa objetiva-se pesquisar o processo social no contexto em que o mesmo ocorre e envolve observação, descrição, compreensão e significado de um fenômeno, bem como hipóteses construídas após observação e não pré-concebidas.
} 
1) percep̧̧ão e apreensão - envolve a percepção no reconhecimento da situação-problema e apreensão na familiarização com o assunto a ser modelado;

2) compreensão e explicação - refere-se a compreensão na formulação do problema, explicitação na formulação do modelo matemático e explicitação na resolução do problema a partir do modelo e;

3) significação e expressão - envolve a significação na interpretação da solução e validação do modelo e a expressão do processo e do resultado.

Nessa prática, os estudantes agruparam-se de acordo com seus interesses (quatro grupos de três estudantes e outro grupo de quatro) para o desenvolvimento e registraram em seus cadernos os apontamentos decididos pelo grupo em cada fase da Modelagem. O professor pesquisador atuou como mediador do processo, conduzindo a prática de acordo com as fases anteriormente descritas. Salienta-se que maior parte da prática foi desenvolvida na sala de aula, sendo realizada em espaço extraclasse somente a coleta de informações complementares, após familiarização inicial com o assunto.

O tema e a situação-problema foram propostos pelo professor pesquisador, visto que a Modelagem na Educação se orienta pelo ensino dos conteúdos (curriculares e não curriculares) e ao mesmo tempo, realização de pesquisa. Ele surgiu devido ao potencial interesse que pode conter, pois a atividade envolve participação ativa na busca de informação e dados pelos próprios estudantes, pois independente de quaisquer características que os estudantes possam ter, todos vão à escola com um calçado. Com orientação para o ensino com base em investigação, o professor pesquisador apresentou o tema da prática com pequeno texto introdutório, ilustrado na Figura 1, o qual foi discutido posteriormente. Na sequência, foi conduzida a prática de acordo com os referentes a cada uma das fases da Modelagem.

Figura 1. Texto apresentado aos estudantes ao iniciar a prática de Modelação

\section{p tamanho do seu calçado}

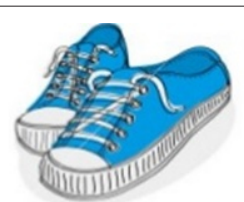

Em todo o mundo existem várias numerações diferentes para um calçado, o que torna complicado comprar sapatos sem os provar. Mas se você optar pela compra na internet em sites estrangeiros? Como escolher corretamente a numeração? Estas e outras questões levam-nos a pensar: quandoe onde iniciou essa história de número de calçado?

Você sabia? ... Tudo começou com um decreto do rei Eduardo I, da Inglaterra, em 1305. Ele estipulou, por exemplo, que trintam e quatro grãos de cevada equivaleriam ao número 34 e assim por diante. Isso facilitou a vida deles e a dos clientes que, antes da padronização, precisavam provar várias vezes um sapato até que ele ficasse pronto. Existem algumas tabelas que permitem determinar o tamanho do seu calçado. No Brasil, a numeração dos calçados varia 13 (bebês) a quase 50 (adulto), com diferença bastante pequenas entre um número e outro.

Mas como saber que é o tamanho adequado para o seu pé, se hoje em dia não temos cevada a nossa disposição com facilidade para realizar a comparação?

Então, como se pode determinar a numeração de um calçado?

Fonte: elaborado pelos autores. 
Os dados para análise foram constituídos mediante materiais produzidos pelos estudantes $\left(\mathrm{E}_{\mathrm{n}}\right)$, gravações em áudio e vídeo e das observações de natureza não estruturada escritas do professor pesquisador. Nesta pesquisa coaduna-se das ideais de Bogdan e Biklen (1994) de que a obtenção dos dados tem ênfase maior no processo em comparação ao produto. Então, para estudar o problema e como se manifesta na prática de Modelagem utilizou-se os procedimentos da análise do discurso de Gill (2002). Inicialmente, tomaram-se os materiais escritos pelos estudantes e fez-se leitura atenta. As falas dos envolvidos foram transcritas e identificaram-se as ideias principais (pontos-chave) comparando-as com os escritos; em seguida, produziram-se notas analíticas no material, apoiadas pela observação do pesquisador, interrogando o texto a partir do problema, do referencial teórico e das pesquisas similares já desenvolvidas. Realizou-se a codificação, procurando ver o discurso a partir do sentido; finalmente a busca de um padrão, do argumento constituído pelo dito e pelo não dito, descrevendo com minúcias os elementos identificados. Em seguida, verificaram-se os resultados obtidos, comparando-os com teoria e resultados de outras pesquisas, sendo redigida, então, a pesquisa.

\section{A prática de Modelagem na Educação - síntese descritiva}

Nesta seção apresenta-se a síntese descritiva da prática elaborada a partir dos materiais produzidos pelos estudantes, e a transcrição da gravação.

A leitura do texto ilustrado na Figura 1 e o destaque à situação-problema apresentada possibilitaram aos estudantes a comparação entre um grão de arroz (pois a cevada não é conhecida na região) com o tamanho do calçado que usavam. As primeiras hipóteses apontadas para a situação-problema, expressas de modo impulsionador, foram: para saber a numeração do calçado "pode ser olhando na caixa" (E3) ${ }^{8}$ ou "a gente experimenta" (E5). A intervenção do pesquisador então direcionou para a percepção de como poderia ser determinada a numeração de um calçado caso não se possuísse oportunidade de prova:

Como poderíamos determinar a numeração de um calçado para qualquer pessoa? Como que eu posso olhar pra um calsado e dizer: este dali é um 35, este outro 39. ... o que cada um pensou? [PP].

Pode ser em centimetros. Mediria o sapato e diria quanto ele vale em centímetro. [E1]. Pode ser um número pra cada idade [E15].

Dos primeiros apontamentos seguidos de reflexão, os estudantes levantaram a hipótese de que a numeração do calçado pudesse ter relação com a idade, posteriormente negada. $\mathrm{Na}$ sequência, optaram por medir, em centímetros, o pé de cada um dos colegas da sala de aula, e não o calçado que estavam usando, estipulando assim uma variável. Partiram das medidas dos pés após contorná-los em folhas de papel: a opção de registro foi pelas medidas de maior comprimento e menor e maior largura (Figura 2).

\footnotetext{
${ }^{8}$ As expressões abreviadas E e PP referem-se à identificação do Estudante e do Professor Pesquisador, respectivamente.
} 
Figura 2. Desenho do pé realizado pelo estudante 7, na prática de Modelação

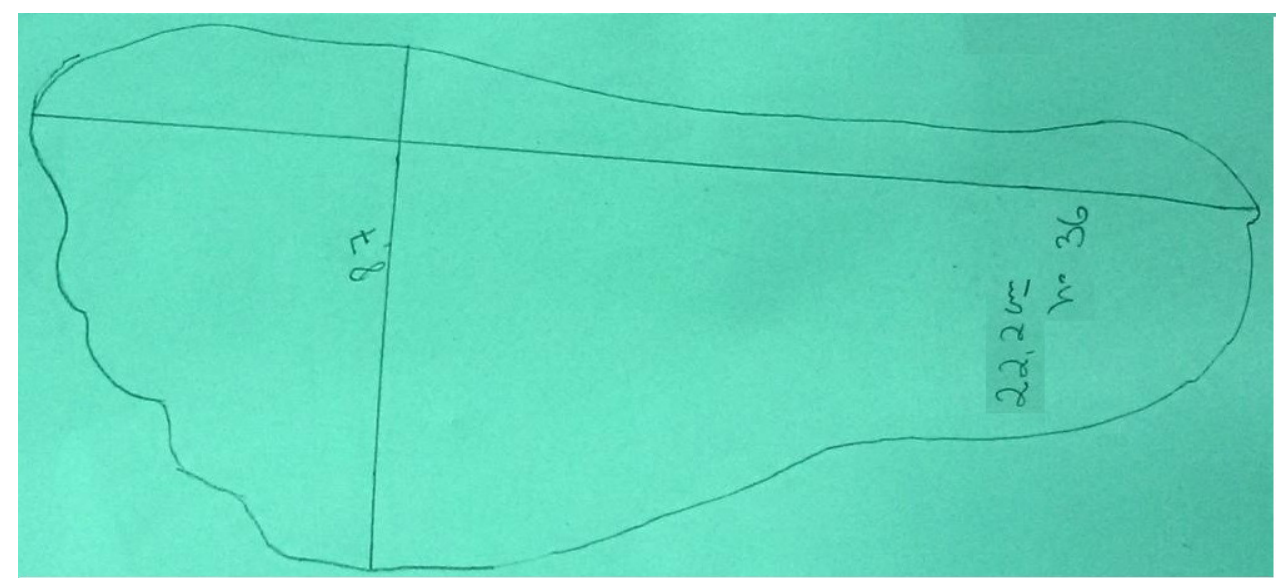

Fonte: Elaborado pelo estudante 7.

Nesta fase da prática foi necessária intervenção do pesquisador para a utilização da régua e registro da medida correspondente. Exploraram-se então noções de medidas de comprimento e números decimais, conceitos ainda não bem claros para os estudantes. Posteriormente, realizaram estudo 'exploratório', extraclasse, trazendo na aula seguinte contribuições para a familiarização com o tema como: tabelas de numeração de calçados sugeridas por empresas, curiosidades e lenda, dentre outras.

A atividade prosseguiu com a escolha de uma das medidas apontadas pelos estudantes na figura, instigados a partir da proposição de PP: "Vamos supor que iremos comprar um calçado, o que é mais importante olhar primeiro: o comprimento ou a largura dele?”. A opção dos estudantes foi pela medida do comprimento do pé e, na discussão do que seria mais provável para encontrar a numeração do calçado, decidiram por registrar as devidas numerações dos calçados que cada um estava utilizando no momento. O registro inicial se deu na linguagem natural, inicialmente em cada folha e, depois, todas compiladas na lousa.

A Tabela 1 expressa medidas organizadas pelo grupo, quando instigados pelo professor pesquisador a "escrever em espaço menor" ou de "forma mais resumida" os dados registrados por eles na lousa. Nesta fase, o pesquisador trabalhou aspectos relacionados ao tratamento de informação, com destaque à representação tabular e gráfica. Isto ocorreu devido à identificação de conceitos equivocados dos estudantes sobre os mesmos, como por exemplo, considerar como tabela algo apenas contendo dados sem identificação e/ou fonte. 
Tabela 1. Levantamento inicial do comprimento do pé (cm) e do número do calçado dos estudantes

\begin{tabular}{cccc}
\hline Comprimento $\mathbf{( c m )}$ & Número do calçado & Comprimento $\mathbf{( c m )}$ & Número do calçado \\
21 & 34 & 22,5 & 36 \\
21,5 & 35 & 19 & 31 \\
22 & 35 & 23,8 & 37 \\
21,5 & 36 & 20 & 33 \\
22,5 & 36 & 24,2 & 38 \\
19,5 & 33 & 23,2 & 37 \\
22 & 35 & 25,4 & 38 \\
19,5 & 33 & 22,2 & 36 \\
\hline
\end{tabular}

Fonte: elaborado pelos autores a partir dos dados da pesquisa.

Na sequência da atividade, a discussão sobre os dados da Tabela 1 possibilitou que os estudantes: (i) identificassem numerações repetidas; (ii) percebessem a existência de comprimentos de pés bastante distantes para uma mesma numeração, por exemplo, a numeração 38 (linhas destacadas na Tabela 1). Tais constatações foram suficientes para que percebessem que algo poderia não estar correto com aqueles dados. Duas foram as opções indicadas pelo grupo: (i) revisar as medidas de comprimento do pé; e (ii) efetuar, novamente, medição e verificar a numeração considerando apenas uma marca de calçado. Posteriormente, obteve-se em um estabelecimento da cidade uma determinada caixa de tênis que continha em uma das suas faces externas a figura representativa da base do calçado com respectiva numeração e comprimento do pé. A partir dela, no encontro seguinte, repetiram a atividade, agora todos com a mesma unidade referencial. Em seguida, registraram e reorganizaram dados conforme Figura 3.

Figura 3. Representação tabular elaborada pelo estudante 8

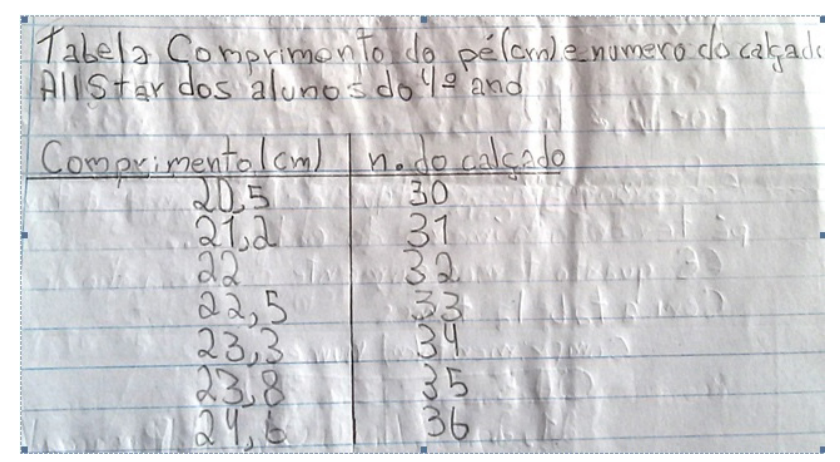

Fonte: Registros do estudante 8.

O estudo dos dados, mediante incentivo do professor pesquisador, possibilitou-os percepção de que 'a variação no comprimento de uma numeração para a seguinte não é cons- 
tante', o que não os permitia indicar o comprimento exato para numeração 37 (na continuação da tabela) e assim obter um modelo. Contudo, aprenderam que tal variação não era uniforme, a cada nova numeração, em média, o comprimento do pé, aumenta por volta de $0,7 \mathrm{~cm}$. Esta percepção e apreensão possibilitou-os determinar a numeração a partir desta variação (Tabela 2), obtendo assim um modelo para resolver o problema.

Tabela 2. Modelo que descreve o comprimento do pé (cm) em função do número do calçado, a partir da menor numeração obtida pelos estudantes

\begin{tabular}{cc}
\hline Comprimento $(\mathbf{c m})$ & Número do calçado \\
\hline$+0,7$ & 30 \\
20,5 & 31 \\
21,2 & 32 \\
22,6 & 33 \\
23,3 & 34 \\
24 & 35 \\
24,7 & 36 \\
25,4 & 37 \\
$\ldots$ & $\ldots$ \\
\hline
\end{tabular}

Fonte: Reelaborado pelos autores a partir dos registros do estudante 1.

$\mathrm{Na}$ validação, ao multiplicarem o valor da numeração por 0,7 , obtiveram resultados próximos aos contidos na Figura 3 (por exemplo: 0,7 x $30=21 \mathrm{~cm}$ (20,5 na tabela), e verificaram a necessidade de reduzir 0,5 do produto encontrado. Desse modo, determinavam modelo capaz de determinar o tamanho do pé, descrito por eles em linguagem natural. No entanto, logo perceberam que este procedimento era útil apenas para determinar o tamanho do pé tendo a numeração do calçado. A pretensão consistia no inverso.

Neste sentido, a atenção voltou-se novamente para a Figura 3, atendo-se agora à variação da numeração. Identificaram então, que para cada aumento de três numerações havia um aumento de dois centímetros no comprimento do pé (Tabela 3 repetindo valores da Figura 3). Tal constatação permitiu que completassem novamente a tabela, a partir da menor numeração (30), primeiro aumentando de dois em dois a medida do comprimento do pé e a numeração correspondente (cor cinza na Tabela 4), indicando o domínio de um padrão recursivo. Depois, preenchendo as outras linhas existentes entre essas (quando identificaram a proporção de que a cada 1 (um) centímetro de aumento na medida do comprimento do pé, a numeração aumentara em 1,5). A partir de então, determinaram outro modelo, que foi validado na sequência (Figura 4). 
Tabela 3. Percepção do Estudante 9 sobre a relação existente entre o comprimento do pé $(\mathrm{cm})$ e o número do calçado dos estudantes constante na Figura 3

\begin{tabular}{|c|c|}
\hline Comprimento $(\mathrm{cm})$ & Número do calçado \\
\hline 20,5 & 30 \\
\hline 21,2 & 31 \\
\hline 22 & 32 \\
\hline 22,5 & 33 \\
\hline 23,3 & 34 \\
\hline 23,8 & 35 \\
\hline
\end{tabular}

Fonte: Reelaborado pelos autores com base nos registros do estudante 9 .

Tabela 4. Modelo que descreve a numeração do calçado em função do comprimento do pé (cm)

\begin{tabular}{|c|c|}
\hline Comprimento (cm) & Número do calcado \\
\hline 20,5 & 30 \\
\hline 21,5 & 31,5 \\
\hline 22,5 & 33 \\
\hline 23,5 & 34,5 \\
\hline 24,5 & 36 \\
\hline 25,5 & 37,5 \\
\hline 26,5 & 39 \\
\hline
\end{tabular}

Fonte: Elaborado pelos autores a partir de dados da pesquisa.

A solução à situação-problema apresentada na Figura 4 ilustra, em linguagem natural e aritmética, o reconhecimento de regularidade e generalização para a situação, sendo expresso desta forma o pensamento algébrico em linguagem diferente da simbólica abstrata. A resolução a partir do modelo indica que os estudantes têm a compreensão de que para determinar a numeração necessitam multiplicar por 1,5 a medida do comprimento do pé, e então reduzir uma unidade deste valor.

No final da atividade realizaram validação do modelo obtido quando cada estudante verificou se seu comprimento de pé correspondia à numeração encontrada por meio do modelo. Outras ações constantes nesta etapa da Modelagem referiram-se à organização do material escrito no caderno e à divulgação do trabalho realizado aos demais colegas da escola. Além disso, duas estudantes realizaram a socialização de sua visão da atividade em mostra municipal, regional e estadual, efetivando assim a realização da etapa de expressão contida na terceira fase do processo de Modelagem, de acordo com Biembengut (2014). 
Figura 4. Resolução do problema e validação do modelo realizada por um grupo de 4 estudantes

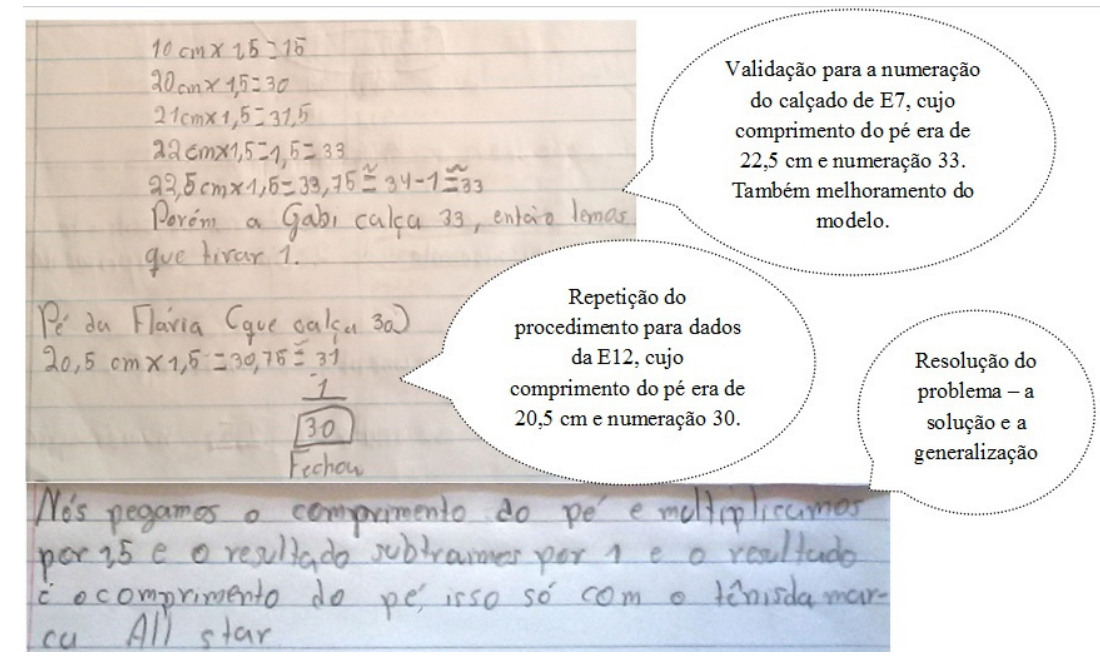

Fonte: Registros dos estudantes 7 e 9.

\section{Discussão dos resultados}

Essa pesquisa desenvolvida a partir de prática de Modelagem na Educação, com 16 estudantes dos Anos Iniciais da Educação Básica, pretendeu compreender e analisar como eles resolvem situações-problema, cujos modelos podem exigir linguagem simbólica e como os expressam em termos de utilização de linguagens. Similarmente ao estudo de English (2010) e English e Waltters (2004a, 2004b), os estudantes apresentam abordagens para resolução da situação-problema, não se restringindo apenas a um mapeamento de dados. No processo de resolução realizado evidenciou-se o processo cíclico das etapas da Modelagem, ou seja, entendeu-se que os alunos: percebem e apreendem; interpretam, compreendem e explicitam e; validam constantemente as informações e dados relativos ao problema. Ademais, apresentam argumentos para a tomada de decisão.

A prática proporcionou aos estudantes a exploração e a manipulação de quantidades e identificação de uma relação entre a numeração e o comprimento do pé, obtendo generalização de maior complexidade do que as evidenciadas nos estudos de Tortola (2012) ou em Fiorentini, Fernandes e Cristovão (2005) - anterior ao $7^{\circ}$ ano. Ao estabelecerem a generalização, identificaram taxa de variação (tabelas 2 e 3), quarto item recomendado pelo National Council of Teacher of Mathematics (2000) para o ensino de álgebra referente ao nível de ensino em que os estudantes se encontram, ilustrado no Quadro 1. Ademais, ao concluírem que valores próximos de comprimentos dos pés teriam mesma numeração para o calçado e não apenas um tamanho específico, deixaram transparecer a significação do modelo encontrado, denotando desta forma a constante percepção, compreensão, validação e reflexão a respeito das hipóteses levantadas, bem como das estratégias utilizadas e as consequências das ações durante o descobrir 
da numeração do calçado. Ilustraram, desta forma, o pensamento crítico e o desenvolvimento cognitivo proporcionados pela Modelagem.

Desta prática de Modelagem, destacam-se apontamentos reflexivos dos estudantes durante a busca por solução: (i) de que a numeração de um modelo de calçado pode variar de uma fábrica para outra, pois existem comprimentos de calçados diferentes para a mesma numeração; (ii) indicam que uma determinada numeração, por exemplo, 30, refere-se a um intervalo de duas medidas (por exemplo, $24,4 \mathrm{~cm}$ a $25 \mathrm{~cm}$ ) e não apenas a um valor fixo. Isto indica que o processo de Modelagem requereu dos estudantes a significação da matemática (item ii) e do contexto (item i) envolvidos no estudo do problema, ou seja, a ideia elementar de intervalo. Em função desta constatação indicaram que teriam um valor próximo para a numeração do calçado e não uma numeração diferente para cada comprimento. Tais apontamentos para a situação-problema ilustram que a Modelagem com problemas reais vai além do simples levantamento de dados sem constante interpretação, compreensão e validação, como destacado por English (2010). Oferece oportunidade para que os estudantes, ao significarem a situação estudada, possam modelar matematicamente da forma que seja mais significativa para eles.

Outro destaque emergente da prática refere-se à naturalidade apresentada pelos estudantes durante o processo de resolução, ao apontarem hipóteses e testá-las em seguida, validando as respostas ou o encontrado. Tudo isso, de modo espontâneo. Tal fato não ocorria com tanta naturalidade no início da primeira prática. Os estudantes se sentiam encorajados para apontarem caminhos ou estratégias a serem realizadas, mencionando apontamentos nem sempre válidos ao final da discussão. Durante a resolução da situação-problema tornou-se natural o processo de existir um problema, indicar hipóteses e testá-las para validação. A realização da prática permite inferir que estudantes de Anos Iniciais da Educação Básica são receptivos às atividades de Modelagem na Educação, participam, efetivamente, de forma comprometida, demonstrando entusiasmo nas ações, diferentemente, dos apontamentos que justificaram a pesquisa de Luna, Souza e Santiago (2009) de que os estudantes podem ser passivos e apresentarem resistência à postura investigativa.

A resolução da situação-problema com a obtenção do modelo ilustra o tipo de linguagem de domínio dos estudantes, aquela a que estão mais familiarizados. Mesmo não dominando o campo algébrico do modo formal (linguagem algébrica simbólica), os estudantes resolveram o problema evidenciando 'domínio informal' ao completar a tabela (modelo), característica do pensamento algébrico anterior à linguagem simbólica (FIORENTINI; FERNANDES; CRISTÓVÃO, 2005). De acordo com Kieran (2004), esta forma de pensar e expressar compreensões, particularidade do pensamento algébrico nos Anos Iniciais, pode ser realizada sem a linguagem simbólica. Os estudantes utilizaram registros variados para a expressão do modelo (a generalização) e a resolução do problema, com destaque à linguagem natural e à aritmética tabular, indicativos do referido pensamento para este nível de ensino (CARRAHER; SCHLIEMANN, 2007; KIERAN, 2004). Ao fazerem uso de representações múltiplas, os estudantes têm facilitado o processo de organizar e comunicar os seus pensamentos e de acordo com o National Council of Teacher of Mathematics (2000), isso é indispensável no processo de aprendizagem.

Representações variadas, incentivadas pelo professor, além de exprimir o modelo, também enriquecem e aprofundam o raciocínio algébrico (KAPUT, 2008). O destaque à representação natural do modelo, no momento inicial é possível, pois de acordo com Duval (2003), a linguagem natural é um registro de representação semiótica discursivo, não algorit- 
mizável, empregado nas associações verbais, nas formas de raciocinar, nas deduções válidas a partir de definições. A solução ao problema registrado com uso de linguagem natural reflete as compreensões dos estudantes na busca de generalizações e formalização do pensamento desenvolvido com seus cálculos. Os estudantes possuem então, capacidade de generalizar e estabelecer relações numéricas, desenvolvendo também a capacidade de fazer previsões como expressou E5 [...] Assim, posso achar qual o calçado para alguém que tem $28 \mathrm{~cm}$ de comprimento. Desta forma, as ações dos estudantes atendem às características do pensamento algébrico descritas por Blanton e Kaput (2005).

English (2010) destaca que estudantes deste nível de ensino, em atividades de Modelagem, obtêm desenhos, esquemas, textos, tabelas, gráficos de barras que se constituem modelos matemáticos, solução de situações-problema apontadas. Apesar de não terem pensamento algébrico formal desenvolvido, os estudantes desta pesquisa revelaram ao elaborar os modelos, aspectos característicos do domínio natural destes ao proporem os mesmos em linguagem natural, subsidiados pelo aritmético e algébrico informal, com apoio de esquemas e quadros. As tabelas e quadros utilizados pelos estudantes constituem-se em instrumentos importantes para o pensamento algébrico, pois, de acordo com Smith (2008), estes possibilitam registros organizados dos dados obtidos e facilitam a apreciação dos mesmos, dando ênfase à relação entre as variáveis envolvidas. Nesta pesquisa, os estudantes utilizaram durante o processo diferentes registros de representação para a expressão do modelo, não estando sempre no registro natural ou numérico. Desta forma, percebeu-se a resolução do problema com modelos que extrapolam os obtidos por estudantes deste nível de ensino nos estudos de Tortola (2012), quando registrou a presença de desenhos, paralelamente à produção escrita e oral das crianças, e de Almeida e Tortola (2014) com modelos expressos na linguagem natural e numérica.

A partir desta análise pode-se inferir que práticas de Modelagem propiciam a estudantes do $4^{\circ}$ Ano do Ensino Fundamental resolver situações-problema desenvolvendo "formas de pensar no âmbito das atividades" (KIERAN, 2004, p. 149), as quais podem ser desprovidas de linguagem simbólica para a formulação do modelo. Um problema que pode requerer domínio simbólico, nesta experiência mostrou ser passível de solução com uso de linguagem pré-simbólica, uma vez que os estudantes utilizam várias formas de pensar e vários tipos de linguagem no processo, as quais subsidiam respostas plausíveis ao problema. Isto porque "analisar relações entre quantidades, observar a estrutura, estudar variações, generalizar, resolver problemas, modelar, justificar, provar e prever" (KIERAN, 2004, p. 149) são ações que evidenciam pensamento algébrico neste nível de escolaridade. Porém, não requerem necessariamente domínio simbólico. Portanto, a linguagem simbólica só será utilizada na expressão de modelos que resolvem o problema se seu uso for incentivado pelo professor para que se 'escreva mais pequenino, mais resumidinho'.

\section{Considerações finais}

Nesta pesquisa pretendeu-se compreender e analisar como estudantes dos Anos Iniciais, em atividade de Modelagem, resolvem situações-problema que podem requerer domínio algébrico simbólico. A partir da compreensão identificaram-se as linguagens utilizadas nas diferentes expressões do modelo. Neste sentido, a partir da análise dos dados, evidenciou-se 
que estudantes do $4^{\circ}$ ano do Ensino Fundamental resolvem as referidas situações: (i) inicialmente, a partir de seus próprios conhecimentos e da forma mais significativa para eles. Criam significados, expressão do pensamento a partir da utilização de linguagem natural e recursos (como figuras e esquemas) e; (ii) posteriormente, quando incentivados, estabelecem relações e atribuem significados a partir do que já sabem, e generalizam, ampliado para a utilização de quadros, tabelas e expressões pré-simbólicas ou narrativizadas, quer dizer, com formas mais elaboradas e adequadas para a idade. Neste processo de Modelagem, eles generalizaram, argumentaram e expressaram o modelo que propiciou a resolução do problema, possibilitando também o aprendizado da álgebra. Ao fazer uso destas representações múltiplas do modelo, os estudantes tiveram facilitadas e ampliadas as formas de organizar e expressar seus pensamentos e também de enriquecer a aprofundar o pensamento algébrico.

No processo de resolução de situação-problema, ao proporem modelos, os estudantes estabeleceram relações numéricas entre as duas variáveis envolvidas - numeração do sapato e comprimento do pé - expressando-as na forma tabular subsidiada pela linguagem natural. Em seguida, elaboraram generalização/modelo (abstrata e complexa para o nível) solucionando o problema, expressando o modelo de formas distintas, ou seja, com vários registros de representação/linguagens - linguagem natural -, subsidiado pelo domínio aritmético e algébrico informal ou pré-simbólico, apoiada por esquemas, tabelas e quadros; utilizaram de estratégias com base aritmética para descrever padrões e/ou regularidades e a partir dela fazem previsões (como no caso de uma numeração de sapato maior que a deles), as quais serão explicadas e argumentadas. Tem-se assim a representação de processos racionais perpassados ao longo do processo de compreensão: os significados. Um processo semiótico de entender por meio dos modelos e não propriamente apenas os modelos obtidos, inferindo maior importância ao que aprendeu por meio da educação algébrica do que dela própria.

Após estas constatações ao longo do processo, verificou-se que os estudantes dos Anos Iniciais que resolvem situações-problema com Modelagem, atingem os objetivos propostos pelo National Council of Teacher of Mathematics (2000) para o ensino de Álgebra referente ao nível, e extrapolam para os objetivos dos Anos Finais ao descreverem a relação existente entre o comprimento do pé e a numeração de um calçado, utilizando-se dos modelos para realizar projeções. Portanto, a Modelagem contribui para a iniciação do desenvolvimento do pensamento algébrico dos estudantes nos Anos Iniciais de acordo com as características de Blanton e Kaput (2005), em especial da vertente pensamento funcional e aritmética generalizada. Ela propicia atividades que exigem maior nível de abstração do que apenas trabalhar com padrões. Desta forma, abordagens como essa contribuirão para sustentar a aprendizagem da Álgebra nos anos seguintes, pois viabilizam no processo a utilização de diferentes linguagens e mais apropriadas na resolução de situações-problema e com destaque maior a semântica-pragmática que a sintaxe.

Com o desenvolvimento da prática de Modelagem constatou-se, também, que professores e estudantes têm tarefas e ações que diferem de outro tipo de atividade desenvolvida, principalmente as características de um ensino tradicional: (i) do estudante - o envolvimento na atividade desafiadora ilustra o potencial que este tipo de prática investigativa oportuniza: percepção, organização, reflexão, capacidade de trabalho em grupo, flexibilidade de ações, cooperação, dentre outros; (ii) do professor - exige que ele atue como mediador, que pergunte mais do que dê respostas; de alguém que aceite o desafio de trabalhar com atividade sem procedimento fixo; tenha planejamento como rotina; flexibilidade para o redirecionamento de perpassar pelas várias 
fases; comprometimento como o ofício da profissão; espírito para aventurar-se com práticas que nem sempre estão estruturadas e que a princípio já se tenha o resultado que os estudantes terão para o problema. Portanto em práticas de Modelagem na Educação, os desafios identificados por Canavarro (2007) para o desenvolvimento do pensamento algébrico nos Anos Iniciais são superados, pois nesta prática de Modelagem o professor: (i) apostou no raciocínio dos estudantes; (ii) acreditou na possibilidade de construção de conhecimento matemático; (iii) proporcionou atividades para o desenvolvimento algébrico ao apostar na Modelagem como prática de ensino com pesquisa ao propiciar discussão, confronto de ideias, argumentações e elaboração de generalizações.

O pesquisador e os estudantes se envolveram ativamente na prática, embora, como se destacou anteriormente, os estudantes ainda não haviam tido contato com a Modelagem. A falta de familiaridade com atividades investigativas pode se constituir em um limitador para o desenvolvimento da prática de Modelagem, fato este que pode ter interferido nos resultados. Outra limitação foi o curto período oportunizado ao pesquisador e mediador das práticas, que não era o professor da turma, para a realização das mesmas. Ademais, pode ser que práticas desenvolvidas por professores regentes tenham resultados diferentes e até melhores que os obtidos, pois os estudantes podem elevar o nível de participação na atividade. Este fato merece ser investigado futuramente. Também se considera interessante investigar se estes estudantes, na continuação de sua vida escolar, apresentam melhores resultados na aprendizagem algébrica interrelacionada aos outros campos da Matemática, em práticas de Modelagem, que outros que não as têm.

Com relação às implicações educativas, destaca-se que as crianças dos Anos Iniciais que se envolvem em atividades de Modelagem com situações-problema de seu contexto e coletam seus próprios dados, têm o processo facilitado para a obtenção do modelo, extrapolando os objetivos relativos ao pensamento algébrico para o ano escolar. Portanto, devem-se oportunizar aos demais estudantes deste nível de ensino tais atividades. Isto implica que Early Álgebra e Modelagem na Educação sejam, realmente, incluídas nos currículos de toda Educação Básica no Brasil, não apenas nos documentos oficiais. Também, que cursos de formação inicial e continuada se constituam em um espaço para o aprimoramento de práticas e reflexões educativas de modo que o método para práticas investigativas sejam constantes nas salas de aula desde o início da Educação Escolar.

\section{Agradecimentos}

À Coordenação de Aperfeiçoamento de Pessoal de Nível Superior (Capes), pela bolsa concedida à primeira autora deste artigo (Processo: BEX 7056-15-0). 
Scheller, M.; Bonotto, D. L.; Madruga, Z. E. F.; Biembengut, M. S.; Sánchez, J. M. C.

\section{Referências}

ALMEIDA, M. W. L.; TORTOLA, E. Modelagem matemática no ensino fundamental: a linguagem de alunos como foco de análise. Jornal Internacional de Estudos em Educação Matemática, São Paulo, v. 7, n. 1, p. 111-142, 2014. Disponível em: <http:// pgsskroton.com.br/seer/index.php/jieem/article/view/88>. Acesso em: 6 dez. 2016.

BEDNARZ, N.; JANVIER, B. Emergence and development of algebra as a problem-solving tool: continuities and discontinuites with arithmetic. In: BEDNARZ, N.; KIERAN, C.; LEE, L. (Ed.). Approaches to algebra: perspectives for research and teaching. Dordrecht: Kluwer, 1996. p. 115-136.

BIEMBENGUT, M. S. Modelagem matemática \& implicações no ensino e na aprendizagem de matemática. 2. ed. Blumenau: Edifurb, 2004.

Modelagem no ensino fundamental. Blumenau: Edifurb, 2014.

. Modelling and applications in primary education. In: BLUM, W. et al. (Ed.).

Modelling and applications in mathematics education: discussion document. New York: Springer, 2007. p. 451-456.

BLANTON, M.; KAPUT, J. Characterizing a classroom practice that promotes algebraic reasoning. Journal for Research in Mathematics Education, Reston, v. 5, n. 36, p. 412446, 2005.

BOGDAN, R. C.; BIKLEN, S. K. Investigação qualitativa em educação: uma introdução à teoria e aos métodos. Lisboa: Porto Editora, 1994.

BRASIL. Ministério da Educação. Diretrizes curriculares nacionais da educação básica. Brasília, 2013.

Elementos conceituais e metodológicos para os direitos de aprendizagem e desenvolvimento do ciclo de alfabetização $\left(1^{\circ}, 2^{\circ}\right.$ e $3^{\circ}$ anos) do ensino fundamental. Brasília, 2012.

. Parâmetros curriculares nacionais: matemática. Brasília, 1998.

Parâmetros curriculares nacionais (1ª a $4^{\mathbf{a}}$ série): matemática. Brasília, 1997.

CANAVARRO, A. P. O pensamento algébrico na aprendizagem da matemática nos primeiros anos. Quadrante, Lisboa, v. 16, n, 2, p. 81-118, 2007.

CARPENTER, T. P.; ROMBERG, T. A. Powerful practices in mathematics \& science: research-based practices for teaching and learning. Madison: University of Wisconsin, 2004.

CARRAHER, D. W.; SCHLIEMANN, A. D. Early algebra and algebraic reasoning. In: LESTER, F. K. (Ed.). Second handbook of research on mathematics teaching and learning. Charlotte: NCTM \& Information Age Publishing, 2007. p. 669-705.

CARRAHER, D. W.; MARTINEZ, M. V.; SCHLIEMANN, A. D. Early algebra and mathematical generalization. ZDM, Heidelberg, v. 40, n. 1, p. 3-22, 2008. 
Modelagem nos anos iniciais da educação básica: ...

CARRAHER, D. W.; SCHLIEMANN, A.; BRIZUELA, B. M. Algebra in the early grades. Hands On!, Cambridge, v. 24, n. 1, p. 8-11, 2001. Disponível em: < http://ase.tufts.edu/ education/earlyalgebra/publications/2001/earlyGrades.pdf>. Acesso em: 05 dez. 2015.

DENZIN, N. K.; LINCOLN, Y. S. (Ed.). Handbook of qualitative research. Thousand Oaks: Sage, 1994.

DUVAL, R. Registros de representações semióticas e funcionamento cognitivo da compreensão em matemática. In: MACHADO, S. D. A. (Org.). Aprendizagem em matemática: registros de representação semiótica. Campinas: Papirus, 2003. p. 11-33.

ENGLISH, L. D. Complex modelling in the primary and middle school years: an interdisciplinary approach. In: STILLMAN, G. A. et al. (Ed.). Teaching mathematical modelling: connecting to research and practice. Dordrecht: Springer, 2013. p. 491-505.

. Mathematical modeling in the primary school: children's construction of a consumer guide. Educational Studies in Mathematics, Dordrecht, v. 63, n. 3, p. 303-323, 2006.

. Modeling with complex data in the primary school. In: LESH, R. et al. (Ed.).

Modeling students' mathematical modeling competencies: ICTMA 13. New York: Springer, 2010. p. 287-300.

ENGLISH, L.; SRIRAMAN, B. Problem solving for the 21st century. In: SRIRAMAN B.; ENGLISH, L. D. (Ed.). Theories of mathematics education: seeking new frontiers. Berlin: Springer, 2010. p. 263-290.

ENGLISH, L.; WATTERS, J. Mathematical modelling with young children. In: HØINES, M. J.; FUGLESTAD, A. B. (Ed.). Proceedings of the 28th Conference of the International Group for the Psychology of Mathematics Education. Bergen: IGPME, 2004a. v. 2, p. 335-342. Disponível em: <http://www.kurims.kyoto-u.ac.jp/EMIS/proceedings/PME28/ RR/RR142_English.pdf >. Acesso em: 1 dez. 2016.

Mathematical modeling in the early school years. Mathematics Education

Research Journal, Dordrecht, v. 16, n. 3, p. 59-80, 2004b. Disponível em: < http://eprints. qut.edu.au/1581/1/1581.pdf>. Acesso em: 1 dez. 2016.

FALCÃO, J. T. R. Alfabetização algébrica nas séries iniciais: como começar? Boletim GEPEM, Rio de Janeiro, n. 42, 2003. p. 27-36. Disponível em: < https://profvinibeck.files. wordpress.com/2014/12/2003-falcc3a3o.pdf>. Acesso em: 1 dez. 2016.

FIORENTINI, D.; FERNANDES, F. L. P.; CRISTOVÃO, E. M. Um estudo das potencialidades pedagógicas das investigações matemáticas no desenvolvimento do pensamento algébrico. In: SEMINÁRIO LUSO-BRASILEIRO DE INVESTIGAÇÕES MATEMÁTICAS NO CURRÍCULO E NA FORMAÇÃO DO PROFESSOR, 2005, Lisboa. Anais... Lisboa: Universidade de Lisboa, 2005. 
Scheller, M.; Bonotto, D. L.; Madruga, Z. E. F.; Biembengut, M. S.; Sánchez, J. M. C.

FIORENTINI, D.; MIORIM, M. A.; MIGUEL, A. Contribuição para um repensar... a educação algébrica elementar. Pro-Posições, Campinas, v. 4, n. 1, p. 78-91, 1993. Disponível em: <http://www.proposicoes.fe.unicamp.br/proposicoes/textos/10-artigos-fiorentinid_ etal.pdf>. Acesso em: 25 jan. 2016.

GILL, R. Análise de discurso. In: BAUER, M. W.; GASKELL, G. Pesquisa qualitativa com texto, imagem e som: um manual prático. 3. ed. Petrópolis: Vozes, 2002. p. 244-270.

GOMES, M. C. V. Álgebra, geometria e aritmética de mãos dadas no ensino fundamental. Boletim GEPEM, Rio de Janeiro, n. 42, p. 47-59, 2003.

KAPUT, J. What is algebra? What is algebraic reasoning? In: KAPUT, J.; CARRAHER, D.; BLANTON, M. (Ed.). Algebra in the early grades. New York: Lawrence Erlbaum, 2008. p. $5-17$.

KAPUT, J.; CARRAHER, D.; BLANTON, M. (Ed.). Algebra in the early grades. New York: Lawrence Erlbaum, 2008.

KIERAN, C. Algebraic thinking in the early grades: what is it? The Mathematics Educator, Athens, GA, v. 8, n. 1, p. 139-151, 2004.

LINS, R. C.; GIMENEZ, J. Perspectivas em aritmética e álgebra para o século XXI. Campinas: Papirus, 1997.

LUNA, A. V. A.; ALVES, J. Modelagem matemática: as interações discursivas de crianças da $4^{\mathrm{a}}$ série a partir de um estudo sobre anorexia. In: CONFERENCIA NACIONAL SOBRE MODELAGEM NA EDUCAÇÃO MATEMÁTICA, 5., 2007, Ouro Preto. Anais... Ouro Preto: UFOP, 2007. p. 855-876.

LUNA, A. V. A.; SOUZA, C. C. C. F. Discussões sobre o ensino de álgebra nos anos iniciais do ensino fundamental. Educação Matemática Pesquisa, São Paulo, v. 15, número especial, p. 817-835, 2013.

LUNA, A. V. A.; SOUZA, E. G.; LIMA, L. B. S. Mathematical texts in a mathematical modelling learning environment in primary school. In: STILLMAN, G. A.; BLUM, W.; BIEMBENGUT, M. S. (Ed.). Mathematical modelling in education research and practice: cultural, social and cognitive influences. Berlin: Springer, 2015. p. 535-543.

LUNA, A. V. A.; SOUZA, E. G.; SANTIAGO, A. R. C. M. A modelagem matemática nas séries iniciais: o germén da criticidade. Alexandria: revista de educação em ciência e tecnologia, Florianópolis, v. 2, n. 2, 135-157, jul. 2009.

MAAß, K. Barriers and opportunities for the integration of modelling in mathematics classes: results of an empirical study. Teaching Mathematics and Its Application, Oxford, v. 24, n. 2-3, p. 61-74, 2005.

NATIONAL COUNCIL OF TEACHER OF MATHEMATICS. Princípios e normas para a matemática escolar. Lisboa: Associação de Professores de Matemática e Instituto de Inovação Educacional, 2007. 
Modelagem nos anos iniciais da educação básica: ...

Principles and standards for school mathematics. Reston: NCTM, 2000.

SCHLIEMANN, A. D.; CARRAHER, D. W.; BRIZUELA, B. M. Bringing out the algebraic character of arithmetic: from children's ideas to classroom practice. New York: Lawrence Erlbaum, 2007.

SILVA, D. P.; SAVIOLI, A. M. P. D. Caracterizações do pensamento algébrico em tarefas realizadas por estudantes do ensino fundamental I. Revista Eletrônica de Educação, São Carlos, v. 6, n. 1, p. 206-222, maio 2012.

SILVA, V.; KLÜBER, T. E. Modelagem matemática nos anos iniciais do ensino fundamental: uma investigação imperativa. Revista Eletrônica de Educação, São Carlos, v. 6, n. 2, p. 228-249, nov. 2012.

SMITH, E. Representational thinking as a framework for introducing functions in the elementary curriculum. In: KAPUT, J.; CARRAHER, D.; BLANTON, M. (Ed.). Algebra in the early grades. New York: Lawrence Erlbaum, 2008. p. 19-56.

SOUZA, E.; LUNA, A. V. A. Modelagem matemática nos anos iniciais: pesquisas, práticas e formação de professores. Revemat: Revista Eletrônica de Educação Matemática, Florianópolis, v. 9, p. 57-73, jul. 2014.

SOUZA, E.; LUNA, A.; LIMA, L. B. S. O papel do professor dos anos iniciais na produção dos discursos das crianças em atividade de modelagem matemática. Boletim GEPEM, Rio de Janeiro, n. 64, p. 34-45, 2014.

TORTOLA, E. Os usos da linguagem em atividades de modelagem matemática nos anos iniciais do ensino fundamental. 2012. 168 f. Dissertação (Mestrado em Ensino de Ciências e Educação Matemática) - Universidade Estadual de Londrina, Londrina, 2012.

TORTOLA, E.; ALMEIDA, L. M. W. Reflexões a respeito do uso da modelagem matemática em aulas nos anos iniciais do ensino fundamental. Revista Brasileira de Estudos

Pedagógicos, Brasília, v. 94, n. 237, p. 619-642, 2013.

VALE, I. et al. Os padrões no ensino e aprendizagem da álgebra. In: VALE, I. et al. (Org.). Números e álgebra. Lisboa: SEM-SPCE, 2007. p. 193-211. 